\title{
ECOC 2013 : un grand écart
}

\author{
Jean-Michel MUR \\ Président du Club optique \\ jm.mur@orange.fr
}

Un grand écart entre ce qui va être - fibres multi-cœurs et photonique sur silicium - présenté lors des conférences, et ce qui est - déploiement des réseaux à $100 \mathrm{Gbit} / \mathrm{s}$ - montré et démontré sur les stands de la partie exposition.

$\mathrm{L}$ a $39^{e}$ édition d'ECOC (European conference and exhibition on optical communications) s'est déroulée du 22 au 26 septembre 2013, à Londres. Elle est restée fidèle à la tradition : une partie conférences prospectiviste et une partie exposition pragmatique. Cependant, il y a désormais un grand écart par rapport à ce qui s'est vu précédemment. En effet, côté conférences, les fibres multi-cœurs étaient à l'honneur et marquent une rupture technologique d'importance par rapport à toutes les fibres optiques installées actuellement. De même, les exposés sur les avancées majeures dans le domaine de la photonique sur silicium donnent un air dépassé aux éléments classiques que sont les composants optiques ou électrooptiques traditionnels. Cela étant, sur les stands, des solutions et des nouveautés étaient proposées pour que les exploitants de réseaux puissent faire face à la demande croissante de débits. Cette croissance est tirée par la nouvelle et quatrième génération de téléphonie mobile - la 4G - et le déploiement de la fibre optique jusqu'à l'habitation des abonnés (FTTH - fiber to the home) avec des débits de $100 \mathrm{Mbit} / \mathrm{s}$ à $1 \mathrm{Git} / \mathrm{s}$ selon les offres commerciales

\section{Quelques extraits de conférences}

\section{Du côté des fibres multi-cœurs}

Côté fibres optiques multi-cœurs, pour Hidehiko Takara, des laboratoires de l'innovation de NTT : « Les récents développements des technologies de transmission basés sur les fibres multi-cours et le multiplexage permettront d'atteindre des capacités de l'ordre du pétabit par seconde par liaison ». Cela étant, chacun $y$ va de ses choix quant au nombre de cœurs. Florilège... Une fibre à 6 cœurs était présentée par les laboratoires des réseaux d'accès de NTT avec l'objectif de tenir dans le diamètre des fibres classiques, soit $125 \mu \mathrm{m}$, tout en limitant les risques de diaphotie (figure 1). Une fibre à 7 cœurs pour les laboratoires de R\&D de KDDI qui vise les transmissions transocéaniques à $100 \mathrm{Tbit} / \mathrm{s}$ et qui offre, grâce à des amplificateurs optiques dotés de 7 cœurs et dopés à l'erbium, une largeur de bande de $5 \mathrm{THz}$. Une fibre optique à 7 cœurs également pour les laboratoires de recherche de NEC qui ont transmis un débit de 128 Gbit/s sur une distance de 1815 kilomètres. Les laboratoires photoniques de NTT et les laboratoires d'optique et électronique de Fujikura, quant à eux, ont travaillé sur un amplificateur à une fibre optique insérée dans une double gaine et comptant 12 cœurs dopés à l'erbium et ytterbium. La connexion avec les fibres du réseau est assurée par une pieuvre de 12 fibres amorces. C'est sur une fibre à 18 cœurs qu'ont été étudiées les caractéristiques de transmission par le département d'ingénierie photonique de I'université technique du Danemark (DTU Fotonik). Le résultat présenté annonçait un débit cumulé de l'ordre du pétabit par seconde. Sans être exhaustif, encore un exemple avec une fibre à 19 cœurs et un prototype d'amplificateur à 19 cœurs dopés à l'erbium, domaine de la présentation de l'institut national japonais de l'information et de la communication et du laboratoire de photonique de Fitel (Furukawa) qui annoncent la faisabilité de transmission sur 900 kilomètres (figure 2).

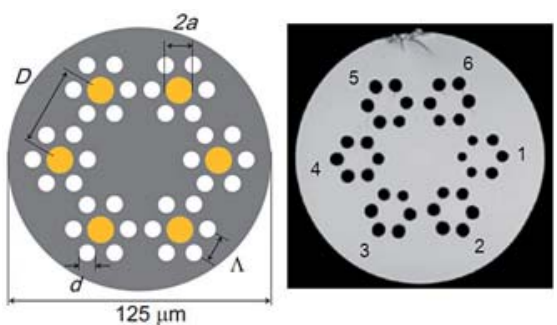

Figure 1. Fibre optique à 6 cœurs entourés par des trous d'air de NTT. Vues en coupe : schéma, à gauche; photo de la fibre, à droite. $D=31,6 \mu \mathrm{m}$; $d=6 \mu m ; 2 a=9,8 \mu m ; \Lambda=10 \mu m$.

Source : Laboratoires des réseaux d'accès $N T$, ECOC 2013.
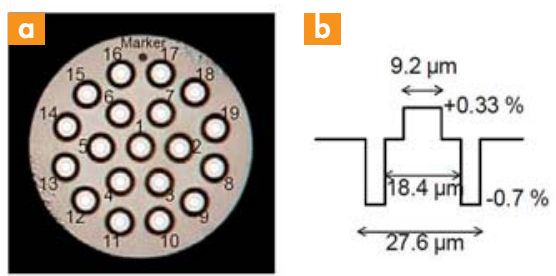

Figure 2. Structure d'une fibre optique à 19 cœurs : (a) vue en coupe, (b) profil des tranchées autour des cœurs.

Source : Furukawa Electric, ECOC 2013.

\section{Du côté des composants}

D'autres conférences traitaient des composants. Extraits... Yu Kurata, des laboratoires de photonique de NTT, présentait les récentes avancées dans l'intégration de semi-conducteurs sur une plateforme en silicium. En exemple, il détaillait les techniques de fabrication et les performances d'un récepteur DP-QPSK intégrant des photodiodes à hautes performances en phosphure d'indium sur un circuit planaire en silicium. Le tout autorisait la démodulation de signaux à 32 Gbauds ( 1 baud = 1 symbole par seconde) (figure 3). De son côté, Jose Capmany, institut de recherche 
de l'université de Valence (Espagne) enchérissait : «Laphotonique sur silicium est une des technologies les plus excitantes et à très forte croissance. Cette technologie est tirée par sa compatibilité avec l'industrie mature des circuits imprimés sur silicium ». Po Dong, des Bell Labs d'Alcatel-Lucent du New Jersey, a présenté les résultats d'une expérimentation de génération, détection et évaluation d'une transmission à $112 \mathrm{Gbit} / \mathrm{s}$ par un circuit intégré photonique sur silicium. En utilisant les techniques de modulation PDMQPSK (polarization division-multiplexed quadrature phase-shift keying), le signal a été transmis sur une distance de 2560 kilomètres sur une fibre optique unimodale standard (figure 4). Il a conclu : «Cette démonstration valide ainsi le fait que les circuits intégrés photoniques sur silicium sont prêts pour les systèmes de transmission en optique cohérente. »

D'autres s'intéressaient aux modulateurs. Ainsi, Robert Palmer, institut de technologie de Karlsruhe, avait démontré expérimentalement le fonctionnement d'un modulateur Mach-Zender jusqu'à 40 Gbit/s. Le chinois Tao Chu, du laboratoire d'optoélectronique intégrée de l'académie des sciences de Pékin, avait élargi ses travaux de recherche à une gamme de débits plus large allant de $20 \mathrm{Gbit} / \mathrm{s}$ à $60 \mathrm{Gbit} / \mathrm{s}$ et a même exposé ses résultats pour un modulateur WDM à $4 \times 50 \mathrm{Gbit} / \mathrm{s}$. II concluait : "Les modulateurs que nous avons développés montrent des solutions prometteuses dans la modulation de la lumière qui pourront être utilisées dans différents domaines de connexions optiques du futur.»

\section{Du côté de l'exposition}

La partie exposition d'ECOC 2013 reprenait les grandes lignes des éditions précédentes à savoir des stands individuels et des zones dédiées. En stands propres aux industriels, la constante vient de la prééminence des entreprises chinoises et, en particulier, celles installées dans la région

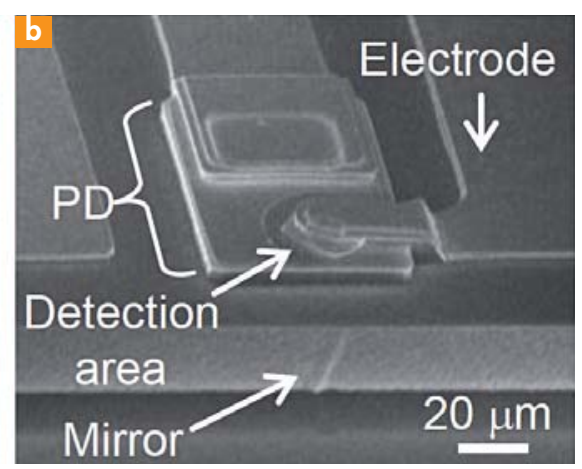

donnaient des conférences pour expliquer leurs vues des différents marchés desservis : large bande, transmission optique à haut débit, réseaux de centres de données, réseaux optiques agiles et intégration optique. Une zone nommée Centre FTTx, vaste espace dans lequel des exposants présentaient

Figure 3. (a) Photographie d'un composant récepteur DP-QPSK. (b) Image d'un micro-miroir et photodiode intégrée. Source : Laboratoire de photonique de NTT, ECOC 2013.

de Shenzhen. En autres représentations, on trouvait des sociétés américaines mais aussi taïwanaises, coréennes et indiennes. L'Europe était bien représentée à travers des industriels de l'Allemagne, de la Belgique, du Royaume-Uni, de la Suisse... mais aussi de la Lituanie et de la Pologne.

Pour nos entreprises nationales, le classique "pavillon France " avait disparu. Heureusement, pour prouver à la communauté internationale des réseaux de communication optiques, que la France existe encore, une dizaine de sociétés étaient là. Un grand merci à 3SPGroup, APEX Technologies, Data-Pixel, Egide, La Précision, Laser 2000, Light Tec, Manlight, Photline Technologies, Vectrawave et Yenista Optics.

Côté zones dédiées, ECOC 2013 a conservé les trois zones habituelles. La zone appelée Market Focus, c'est-à-dire une salle dans laquelle des exposants physiquement leurs solutions et équipements pour les applications FTTB (fibre jusqu'au bâtiment), FTTH (fibre jusqu'à I'habitation), centres de données, etc. le tout avec des démonstrations en direct. Et une troisième zone dédiée à la formation où la société CTTS permettait à tout un chacun de se familiariser avec la mise en œuvre de soudeuses de fibres optiques, I'utilisation de réflectomètres optiques...

\section{Exemples de nouveautés}

Loin d'être exhaustives, quelques nouveautés présentées...

Exfo proposait un nouvel analyseur de spectre optique, le FTB-5230S, qui vise le marché d'une large gamme d'applications de multiplexage en longueur d'onde - CWDM, DWDM, test de réseau hybride, etc. - et un nouvel analyseur de canal optique, le FTB-5230S-OCA, dédié plus particulièrement aux exploitants de réseaux câblés.

Fujikura complétait sa large gamme de soudeuses de fibres optiques avec deux

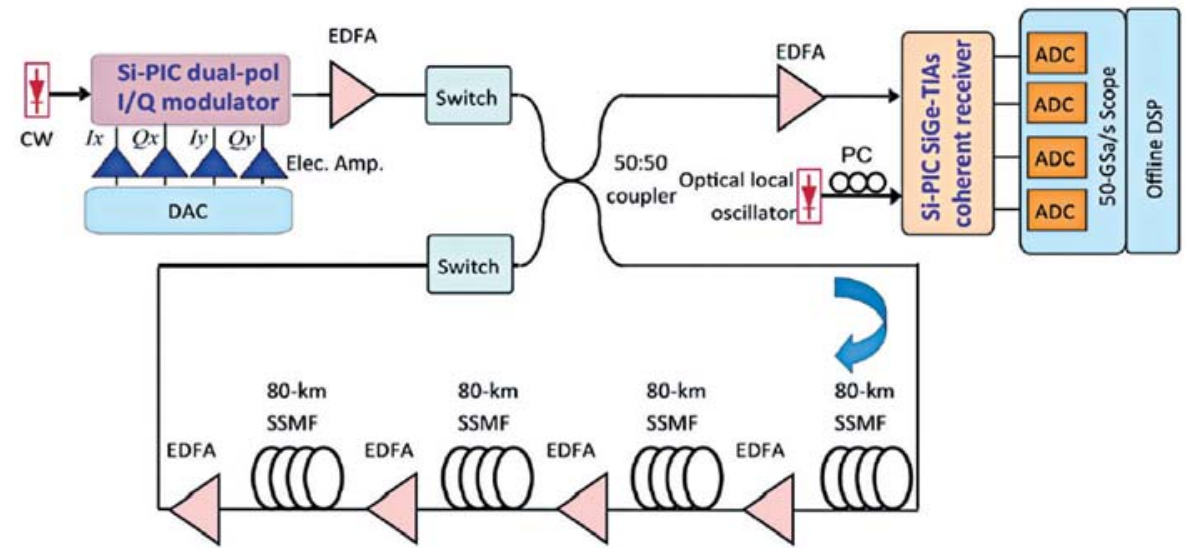

Figure 4. Schéma de l'expérimentation de la génération, détection et évaluation d'une transmission à 112 Gbit/s via un circuit photonique sur silicium. Source : Bell Labs, Alcatel-Lucent, ECOC 2013. 
nouveaux modèles à prix serrés, la 195 pour fibre unitaire et la 19R pour câble en ruban de quatre fibres, ainsi qu'une solution de test dédiée aux centres de données (data center).

Molex présentait un système d'interconnexion de fond de panier optique basé sur la technologie MT offrant la connexion simultanée de 8,12 ou 24 fibres optiques. Cet ensemble a été renforcé pour répondre aux exigences de la norme VITA 66.1. Ainsi, il convient aux applications embarquées dans l'aérospatial, le militaire et le ferroviaire (figure 5).

NeoPhotonics, industriel spécialisé dans les circuits intégrés photoniques (photonic integrated circuit - PIC) offrait toute une nouvelle gamme de composants optiques incluant des récepteurs cohérents à $100 \mathrm{Gbit} / \mathrm{s}$, des lasers accordables à taille réduite, des modulateurs, etc. pour les réseaux à très haut débit, des réseaux optiques passifs (passive optical networkPON) ainsi que des émetteurs-récepteurs pour centre de données. Ces nouveautés répondent aux exigences des réseaux à $100 \mathrm{Gbit} / \mathrm{s}$ mais aussi aux applications à 200 et 400 Gbit/s utilisant des modulations d'ordre élevé comme le 16 QAM (quadrature amplitude modulation).

Sumitomo Electric présentait ses émetteurs-récepteurs optiques pour le 100GBASE-LR4 et se voulait confiant en annonçant, pour fin 2013, les premiers échantillons d'émetteurs-récepteurs optiques répondant aux spécifications du 100GBASE-SR10. Sumitomo s'intéresse

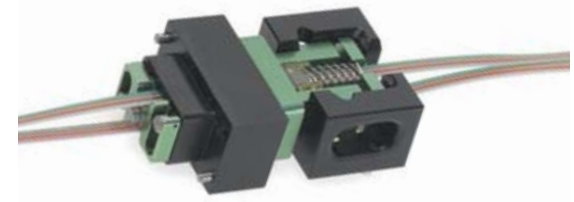

Figure 5. Système renforcé d'interconnexion multifibres pour fond de panier optique à la norme ANSI VITA 66. 1

Source : Molex, ECOC 2013

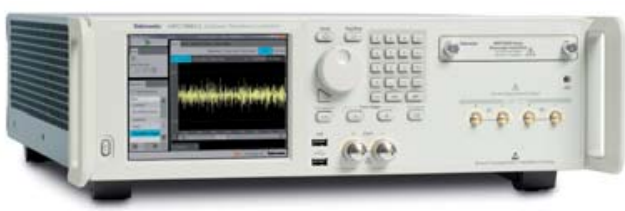

Figure 6. Nouveau générateur de signaux AWG70000. Source : Tektronix, ECOC 2013.

aussi au développement de connectique à haut débit de type QSFP pour le marché des centres de données.

Tektronix exposait un nouveau générateur de signaux - AWG70000 - couvrant une gamme très large allant jusqu'à 20 GHz et dédié aux applications à haut débit. En exemple, son utilisation en laboratoire, par Alcatel-Lucent qui a atteint un débit de 1,5 Tbit/s par la combinaison de huit signauxà 30 Gbauds par canal optique chacun transmettant à 233 Gbit/s (figure 6).

Xilinx, spécialiste des semi-conducteurs, a démontré sur le stand d'interopérabilité de l'OIF (Optical internetworking forum), les capacités de son commutateur IP destiné aux réseaux de transport des paquets optiques et dont la commercialisation a été

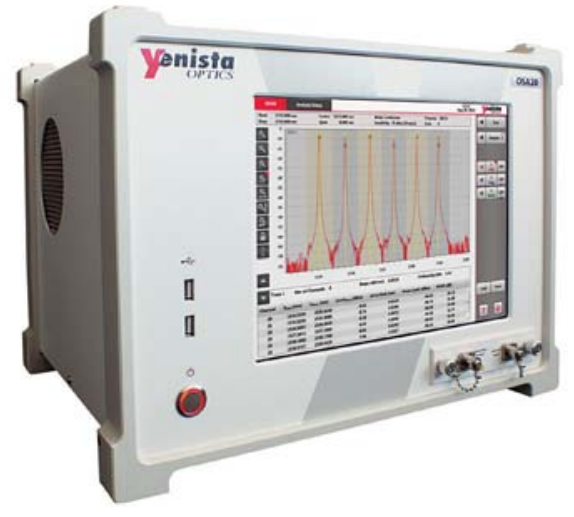

Figure 7. Nouvel analyseur de spectre optique multifonctions - OSA20

Source : Yenista, ECOC 2013.

annoncée lors d'ECOC. À noter que sur le stand de I'OIF, on pouvait voir une dizaine d'autres démonstrations d'interopérabilité entre équipements d'industriels différents (voir encadré).

Yenista présentait un nouvel analyseur de spectre optique - OSA 20 - qui peut servir à la fois d'analyseur de spectre optique classique ou dans d'autres modes d'analyse type laser unimodal ou multimodal, multiplexage en longueur d'onde, source large bande... (figure 7).

\section{À noter}

Vos prochains rendez-vous avec ECOC : à Cannes, pour la $40^{\mathrm{e}}$ édition, du 21 au 25 septembre 2014 (www.ecoc2014.org), puis en Espagne, à Valence, pour la $41^{\mathrm{e}}$ édition du 27 septembre au $1^{\text {er }}$ octobre 2015.

\section{Démonstrations sur le stand OIF}

Les architectures des nouveaux réseaux à $100 \mathrm{Gbit} / \mathrm{s}$ et $400 \mathrm{Gbit} / \mathrm{s}$ et, surtout, l'interopérabilité entre les éléments nécessaires et commercialisés à ce jour, étaient l'attraction sur le stand de I'OIF loptical internetworking forum www. oiforum.com). De fait, plusieurs démonstrations d'interopérabilité étaient installées par divers membres de cette association qui regroupe des industriels des semi-conducteurs, de la connectique et de modules optiques, de systèmes de transport optique ainsi que des fabricants d'appareils de tests et de mesures. Ed Erlan, président du groupe de travail sur I'interopérabilité des couches liaisons et physiques, précisait : "L'OIF a défini une technologie et des caractéristiques pour des liaisons à 25 Gbit/s qui ont été adoptées par l'industrie et qui servent de briques aux applications à 50, 100 et $400 \mathrm{Gbit/s}$. Les démonstrations d'interopérabilité permettent le déblocage des investissements pour les réseaux du futur. » Quelques exemples présentés basés sur les architectures de ces nouveaux réseaux optiques... Quatre démonstrations illustraient les caractéristiques de connexité et d'interfaces aux niveaux physique, électrique, etc. prouvant les transmissions sans erreur dans le cas de liaisons optiques à $100 \mathrm{Gbit/s}$ aux standards 100GBASE-LR4 et 100GBASE-SR. À noter qu'avec la densification des ports et des composants, associée à la puissance électrique nécessaire, un point critique apparaît dans l'exigence d'interopérabilité. II s'agit d'une double capacité pour les équipements : la résistance aux élévations de température et la nécessaire dissipation thermique. D'où, une démonstration sur ce sujet pour des modules optiques de 4 liens à 100 Gbit/s pour le débit de $400 \mathrm{Gbit} / \mathrm{s}$ - ainsi que trois démonstrations sur des liaisons de fonds de paniers optiques.

Les acteurs présents sur le stand de l'OlF étaient les sociétés Amphenol, Applied Micro, Cisco, Finisar, Fujitsu Optical Components, Inphi, Molex, MoSys, Semtech, TE Connectivityet Xilinx, pour les liaisons et systèmes, ainsi qu'Agilent Technologies et Tektronix pour les mesures. 